\title{
Avaliação dos fatores de risco maternos em gestantes admitidas com pré-eclâmpsia grave
}

\section{Evaluation of maternal risk factors in pregnant women admitted with severe preeclampsia}

\author{
Nilce Ariane Spencer Santos ${ }^{1}$. Julio Augusto Alves Gurgel ${ }^{2}$. Carla Gurgel Camurça ${ }^{3}$. \\ 1 Graduada em Medicina pela Universidade Federal do Rio Grande do Norte (UFRN), Residência em Ginecologia e Obstetrícia, \\ Universidade Federal do Ceará (UFC), Fortaleza, Ceará, Brasil. 2 Médico, ginecologista e obstetra com título de especialista pela \\ FEBRASGO, habilitação em ultrassonografia em Ginecologia e Obstetrícia pelo Colégio Brasileiro de Radiologia, Preceptor de \\ Ginecologia e Obstetrícia da Maternidade Escola Assis Chateaubriand (MEAC), Universidade Federal do Ceará (UFC), Fortaleza, \\ Ceará, Brasil. 3 Estudante de Medicina da Faculdade Christus, Fortaleza, Ceará, Brasil
}

\section{RESUMO}

OBJETIVOS: Avaliar os fatores associados ao diagnóstico de pré-eclâmpsia (PE) precoce ou tardia. MATERIAL E MÉTODOS: estudo retrospectivo com 208 gestações com PE na Maternidade Escola Assis Chateaubriand - Universidade Federal do Ceará, no período de um ano (2014 a 2015). O diagnóstico e a gravidade da doença basearam-se nos níveis pressóricos, proteinúria, dados clínicos e laboratoriais. Os fatores de risco considerados foram idade, paridade, histórico de PE em gestação anterior ou na família, níveis pressóricos, dopplervelocimetria. A PE foi considerada pré-eclâmpsia início precoce (PEP) quando diagnosticada antes da $34^{\mathrm{a}}$ semana e pré-eclâmpsia de início tardio (PET) após a 34ª semana. RESULTADOS: Das 208 pacientes analisadas, obtivemos 156 (75\%) casos de PET e 52 (25\%) de PEP. Entre as primíparas (123), 62,8\% eram PET ( $\mathrm{p}=0,06)$. Pacientes que iniciaram o pré-natal com níveis pressóricos mais elevados tiveram maior incidência de PEP. CONCLUSÃO: Dos fatores de risco mais associados com PE grave, observou-se que a Hipertensão arterial crônica foi o mais prevalente; e, dos fatores de risco moderado, a primiparidade, a história prévia de doença hipertensiva em gestação anterior e o Índice Massa Corporal (IMC) elevado foram os mais encontrados. Pacientes que iniciam pré-natal com níveis pressóricos elevados ou hipertensão prévia tem maior risco de desenvolver PEP.

Palavras-chave: Pré-eclâmpsia. Complicações na gravidez. Fatores de risco.

\section{ABSTRACT}

OBJECTIVES: To evaluate the factors associated with the diagnosis of early or late preeclampsia(PE). MATERIALAND METHODS: A retrospective study with 208 pregnancies with PE at the Maternidade Escola Assis Chateaubriand - Universidade Federal do Ceará, in the period of one year (2014 to 2015). The diagnosis and severity of the disease were based on pressure levels, proteinuria, clinical and laboratory data. The risk factors considered were age, parity, history of PE in previous gestation or in the family, blood pressure levels, Doppler velocimetry. PE was considered precocious (PPE) when diagnosed before the $34^{\text {th }}$ week and late (LPE) after the $34^{\text {th }}$ week. RESULTS: Of the 208 patients analyzed, we obtained $156(75 \%)$ cases of LPE and 52 (25\%) of PPE. Among the primiparous (123), 62.8\% were LPE $(\mathrm{p}=0.06)$. Patients who started prenatal care with higher blood pressure levels had a higher incidence of PPE. CONCLUSION: Of the risk factors most associated with severe PE, it was observed that chronic hypertension was the most prevalent; and, of the moderate risk factors, primiparity, previous history of hypertensive disease in previous gestation and high Body Mass Index (BMI) were the most found. Patients who initiate prenatal care with high blood pressure or previous hypertension are at increased risk of developing PEP.

Keywords: Preeclampsia. Pregnancy complications. Risk factors.

Autor correspondente: Nilce Ariane Spencer Santos, Rua Miguel Goncalves, 100, Damas, Fortaleza, Ceará. Telefone: +55 85 99820-6008. E-mail: nilcesantos18@gmail.com

Conflito de interesses: Não há qualquer conflito de interesses por parte de qualquer um dos autores.

Recebido em: 04 Abr 2016; Revisado em: 12 Jun 2016; Aceito em: 27 Jun 2016. 


\section{INTRODUÇÃO}

A Pré-eclâmpsia (PE) é uma doença sistêmica, responsável por 10 a $15 \%$ da mortalidade materno-fetal e em igual proporção ao nascimento de prematuros em todo o mundo. ${ }^{1}$ No Brasil é uma das principais causas de morte materna (37\% das causas de morte obstétricas diretas), o que demonstra a gravidade das complicações decorrentes dessa síndrome hipertensiva. ${ }^{2,3}$

Clinicamente, a pré-eclâmpsia pode ser caracterizada nas formas leve e grave. Esta classificação tem sido amplamente utilizada por basear-se em critérios clínicos objetivos, refletindo no seu prognóstico e conduta durante a gestação. ${ }^{4,5}$ Outra classificação, baseada no momento da instalação da síndrome, divide a PE como pré-eclâmpsia de início precoce (PEP), antes de 34 semanas de gestação, relacionada à falha da migração trofoblástica; e a pré-eclâmpsia de início tardio (PET), depois de 34 semanas, mais associada a características vasculares e metabólicas maternas. ${ }^{4,6-9}$

Atradicionalabordagem de triagemparapré-eclâmpsiaéidentificar os fatores de risco a partir da história materna, classificando pelas características clínicas e demográficas. ${ }^{10}$ No Reino Unido, o National Institute for Health and Clinical Excellence (NICE) emitiu orientações que recomendam que as mulheres devem ser consideradas de alto risco ao desenvolvimento da pré-eclampsia se elas apresentarem pelo menos um fator de alto risco ou dois dos fatores de risco moderado. ${ }^{11}$

Os fatores de alto risco, citados na literatura, são: história de hipertensão em gravidez anterior, doença renal crônica, doença autoimune, diabetes mellitus ou hipertensão crônica; os fatores de risco moderado são a primiparidade, idade maior que 40 anos, intervalo interpartal maior do que 10 anos, o índice de massa corporal (IMC) na primeira visita de $35 \mathrm{~kg} / \mathrm{m}^{2}$ ou história familiar de PE. ${ }^{11}$ Mas esta classificação não distingue qual o tipo de PE, se grave ou leve, ou se tardia ou precoce.

Sabemos que os resultados maternos e perinatais são piores na PEP e, gratificantemente, este é o tipo de PE que pode ser prevenida satisfatoriamente em cerca da metade dos casos com aspirina $100 \mathrm{mg}$ se iniciado antes das 16 semanas, possivelmente por favorecer uma melhor migração trofoblástica. ${ }^{4,12,13}$

A partir de pacientes reconhecidamente de alto risco, pois haviam evoluído para a forma grave de PE, fez-se um estudo retrospectivo para saber se as características maternas encontradas no início do pré-natal coincidem com as citadas pelo estudo NICE e se seria possível classificarmos quais os fatores de risco mais associados à PEP.

\section{MATERIAS E MÉTODOS}

Trata-se de um coorte retrospectivo, onde foram analisados os fatores de risco maternos associados ao diagnóstico de pré-eclâmpsia grave (PEG), tanto de início precoce como de início tardio. Foram coletados dados dos prontuários de pacientes com PE internadas na Maternidade Escola Assis Chateaubriand no período de janeiro 2014 até janeiro 2015. O projeto foi aprovado no Comitê de Ética em Pesquisa da
Maternidade Escola Assis Chateaubriand, em novembro 2014, conforme normas do Conselho Nacional de Saúde.

Os registros destas pacientes foram obtidos através do livro de admissões no Serviço de Medicina Materno Fetal, específica para casos com diagnóstico de pré-eclâmpsia, totalizando 350 prontuários. Seguindo a obtenção dos dados, foi feito um levantamento nos prontuários de pacientes com PEG que preenchiam critérios para tal classificação, totalizando 208 pacientes.

Nos prontuários selecionados foram coletadas características maternas, ultrassonográficas e, estes foram avaliados quanto ao potencial preditivo para os dois tipos de PEG (de início precoce e de início tardio).

As variáveis consideradas na análise foram as seguintes: epidemiológicas (idade, paridade, história de PE em gestação anterior, PE na família, comorbidades, uso de medicação como profilaxia, a exemplo da aspirina e do cálcio, uso de medicação anti-hipertensiva, necessidade de sulfatoterapia na admissão); clínico-biofísicos (peso, altura, pressão arterial (PA) no início da gestação, pressão arterial média (PAM) de admissão). Dados ultrassonográficos (Doppler de artérias uterinas, umbilical e cerebral).

Foram excluídos casos de gemelaridade, malformação fetal e amniorrexe prematura.

Definiu-se pré-eclâmpsia de acordo com os critérios estabelecidos pelo American College of Obstetricians and Gynecologists (ACOG). ${ }^{149}$ Sendo consideradas formas graves (presença de qualquer um dos seguintes sinais e sintomas): PA $\geq 160 \times 110 \mathrm{mmhg}$, proteinuria $>2 \mathrm{~g} / 24 \mathrm{~h}$ ou 2 ou $3+$ no labstick, creatinina $>1,2 \mathrm{mg} \%$, manifestações cerebrais e visuais, edema agudo de pulmão, dor epigástrica, síndrome Hellp. ${ }^{14}$

Classificada como PE precoce quando o diagnóstico é realizado antes de 34 semanas e PE tardia para os casos com diagnóstico a partir das 34 semanas..$^{15-17}$

Para cálculo do tamanho da amostra, foi utilizado o software Raosoft, considerando a prevalência de PE em torno de $6 \%$, nível de significância de $5 \%$, força do teste de $80 \%$ e a margem de erro de $5 \%$. Para determinar a associação entre as variáveis e os tipos de PE, foram utilizados os teste de $t$ de Student para as variáveis quantitativas e os testes qui-quadrado ou exato de Fisher para as variáveis qualitativas. Regressão logística univariada e multivariada foi aplicada a partir dos fatores de risco maternos para tentar obter o poder de predição para PEP ou PET. Os dados foram analisados pelo STATA versão 10.

\section{RESULTADOS}

No período de janeiro de 2014 a janeiro de 2015, selecionouse 208 pacientes admitidas com síndromes hipertensivas e diagnosticadas com PE no serviço de Obstetrícia da Maternidade Escola Assis Chateaubriand para avaliação de fatores de risco (Tabela 1). 
Tabela 1. Distribuição de variáveis maternas quanto ao tipo de PE

\begin{tabular}{|c|c|c|c|}
\hline & $\begin{array}{c}\text { PEP } \\
\mathrm{N}=52\end{array}$ & $\begin{array}{c}\text { PET } \\
\mathrm{N}=156\end{array}$ & $\mathrm{p}$ \\
\hline $\begin{array}{l}\text { IDADE MATERNA, } \\
\text { média } \pm \text { DP }\end{array}$ & $25,9 \pm 6,4$ & $25,7 \pm 7,2$ & $0,712 *$ \\
\hline $\begin{array}{l}\text { ESTATURA (metros), } \\
\text { média } \pm \text { DP }\end{array}$ & $1,5 \pm 0,2$ & $1,53 \pm 0,2$ & $0,851^{*}$ \\
\hline $\begin{array}{l}\text { PESO (gramas), } \\
\text { média } \pm \text { DP }\end{array}$ & $67,7 \pm 17,4$ & $69,5 \pm 17,6$ & $0,483 *$ \\
\hline OBESIDADE, n (\%) & $25(48,1)$ & $74(47,4)$ & $0,382^{\#}$ \\
\hline $\begin{array}{l}\mathrm{IMC}\left(\mathrm{Kg} / \mathrm{m}^{2}\right) \\
\text { média } \pm \mathrm{DP}\end{array}$ & $28,4 \pm 6,8$ & $28,4 \pm 6,8$ & $0,851 *$ \\
\hline PRIMIPARIDADE, n (\%) & $25(48,1)$ & $98(62,8)$ & $0,061^{\#}$ \\
\hline PE PRÉVIA, n (\%) & $20(38,5)$ & $40(25,6)$ & $0,068^{\#}$ \\
\hline ANT FAMILIAR PE, n (\%) & $6(11,5)$ & $20(12,8)$ & $0,880^{\#}$ \\
\hline HAC, n (\%) & $30(57,7)$ & $27(17,3)$ & $0,000^{\#}$ \\
\hline $\begin{array}{l}\mathrm{PAS}(\mathrm{mmHg}) \text { no PN, } \\
\text { média } \pm \mathrm{DP}^{\epsilon}\end{array}$ & $121,2 \pm 16,3$ & $114,0 \pm 14,2$ & $0,006^{*}$ \\
\hline $\begin{array}{l}\mathrm{PAD}(\mathrm{mmHg}) \text { no PN, } \\
\text { média } \pm \mathrm{DP}^{\epsilon}\end{array}$ & $80,9 \pm 13,0$ & $75,4 \pm 11,8$ & $0,010^{*}$ \\
\hline DOENÇA CRÔNICA, n(\%) & $13(25)$ & $31(19,9)$ & $0,407^{\#}$ \\
\hline
\end{tabular}

DP - desvio padrão; PE - pré-eclâmpsia; PEP - pré-eclâmpsia precoce; PET - pré-eclâmpsia tardia; IMC - índice massa corporal; ANT - antecedente; HAC - hipertensão crônica; PN - pré-Natal. / ${ }^{\#}$ teste exato de Fisher, *teste de $t$ de Student.

$\mathrm{OBS}^{\epsilon}=>$ PAS (pressão arterial sistólica) e PAD (pressão arterial diastólica) no início do pré-natal foram dados obtidos em 42 casos de PEP e em 129 casos de PET.

Fonte: elaborada pelos autores

A maioria das pacientes eram primíparas (123), com média de idade de 25 anos (min. 12 e máx. 42), estatura de 1,54 metros (min. 1,41 e máx. 1,75) e IMC de 28,2 (min. 16 e máx. 52). Observou-se que $56 \%$ das pacientes estavam com sobrepeso no início do pré-natal.

A hipertensão foi a morbidade crônica mais frequente, sendo observada em 57 casos $(27,4 \%)$ e esteve associada à primiparidade em $23 \%$ dos casos. Observou-se também a ocorrência de diabetes gestacional, lúpus, cardiopatia, asma, psoríase e epilepsia como doenças crônicas associadas a gestação, porém em quantidade reduzida, não sendo possível associar com os diferentes tipos de PE.

Em relação a complicações maternas próprias das síndromes hipertensivas da gestação, foram constatados 3 casos de descolamento prematuro da placenta, 11 com síndrome Hellp (hemólise, elevação de enzimas hepáticas e plaquetopenia) e 5 casos de eclâmpsia.

Obteve-se nesse estudo 156 casos de PET (75\%) e 52 de PEP (25\%). Ao compararmos estes dois tipos de PE, notouse que não houve diferenças estatisticamente significantes no que se refere à estatura, IMC ou presença de obesidade, idade materna, história familiar e doenças crônicas associadas.
Em 151 pacientes foram encontrados registros pressóricos do primeiro trimestre gestacional, sendo 42 do grupo PEP e 129 do grupo PET. Observou-se que as pacientes que iniciaram o pré-natal com níveis pressóricos mais elevados tiveram maior número de casos de PEP, especialmente quando a PA média estava maior do que $100 \mathrm{mmHG}$ no início da gestação ( $\mathrm{p}=$ 0,03) (Gráfico 1). A elevação da PA diastólica a partir de 100 $\mathrm{mmHg}$ no primeiro trimestre de gestação aumentou o risco da paciente desenvolver PEP mais do que PET ( $O R=1,04$; IC:1,00 a 1,06). Este parâmetro apresentou sensibilidade de $24 \%$ e especificidade de $90 \%$ para uma taxa de $10 \%$ de falso positivo e valor de verossimilhança próximo a 3,0 (Gráfico 2).

Gráfico 1. Box Plot da Relação de PAM quanto aos tipos de pré-eclâmpsia.

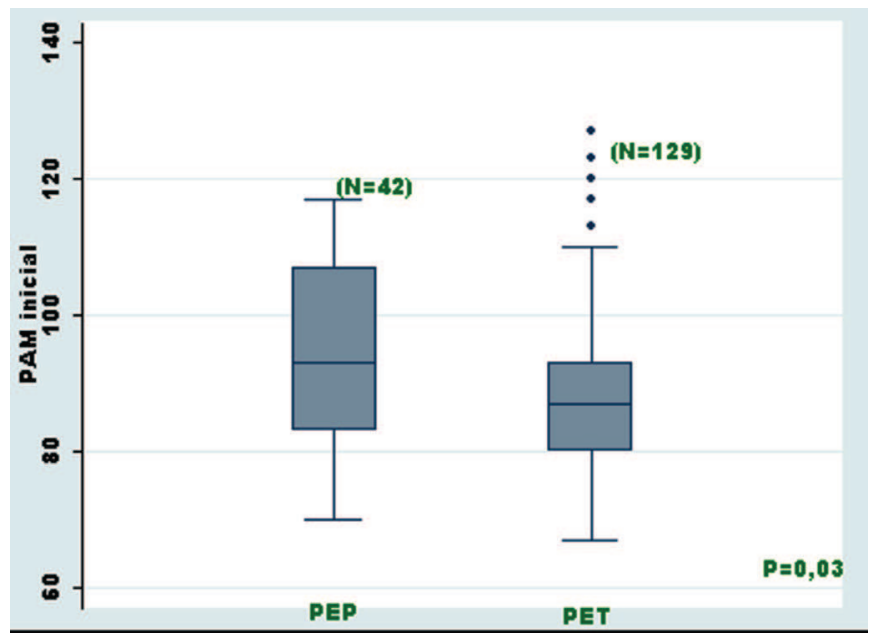

Fonte: elaborado pelos autores

Gráfico 2. Representação da curva ROC (Receiver Operating Characteristic Curve) para a PA diastólica como fator de risco para $\mathrm{PEP}$ entre as pacientes que desenvolveram PE grave.

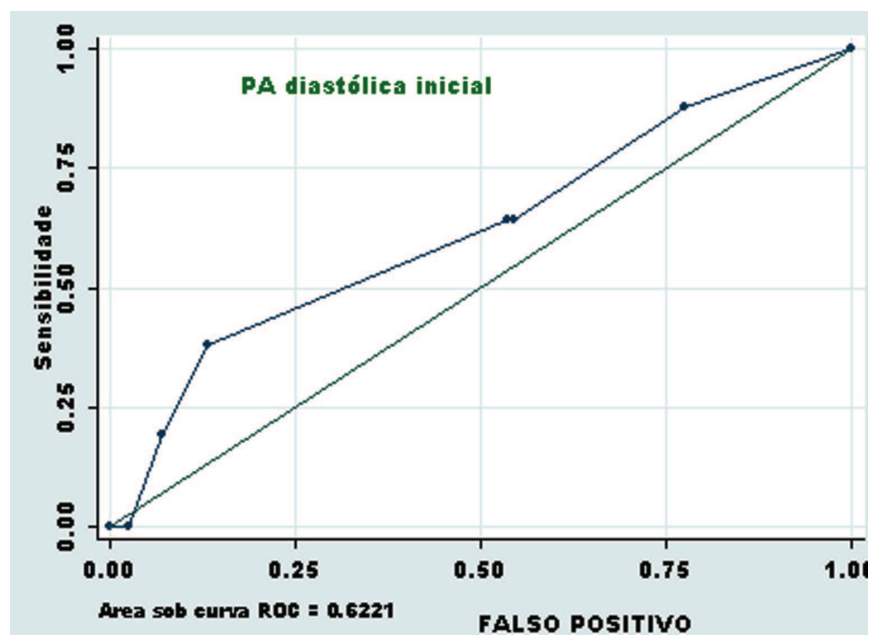

Fonte: elaborado pelos autores

Observou-se que 62,8\% das primíparas haviam evoluído para PET $(p=0,06)$. As demais pacientes, que tinham pelo menos uma paridade prévia, 70\% (60/85) referiram história de doença hipertensiva em gestação anterior, das quais 40 evoluíram para PET e 20 evoluíram para $\operatorname{PEP}(p=0,26)$. 
A história familiar de PE esteve presente em 26 casos (12,5\%) dos casos, e não houve diferença entre os grupos de pacientes que evoluíram para PET e PEP $(\mathrm{p}=0,88)$.

No que se refere à investigação com Doppler, apenas 65 pacientes realizaram a pesquisa de alterações ultrassonográficas, não sendo estes dados estatisticamente significantes para correlacionar com maior prevalência de um desses tipos de PE.

Quanto à profilaxia para pré-eclâmpsia, evidenciou-se que dez pacientes tinham feito uso de aspirina e/ou cálcio. Das que usaram, a maioria desenvolveu PET (8) e não PEP (2), possivelmente melhorando os resultados maternos e perinatais.

\section{DISCUSSÃO}

Observou-se que muitos fatores de risco presentes na assistência materna do Reino Unido não são frequentes no nosso atendimento de rotina do serviço público. Dos fatores de alto risco, confirmamos a importância da história prévia de hipertensão em uma gravidez anterior e a hipertensão arterial crônica. Entretanto, a doença renal crônica, a doença autoimune e o diabetes mellitus foram pouco prevalentes. Quanto aos fatores de risco moderado, a primiparidade e o IMC elevado preponderaram.

A idade materna, a estatura e o peso foram fatores de risco presentes uniformemente entre os dois grupos de PE, sem distinção entre as médias destas variáveis. Pôde-se evidenciar que na população analisada que desenvolveu PEG, 56\% das pacientes encontravam-se com sobrepeso no início do prénatal, o que corrobora com os dados apontados em outros estudos que evidenciam que o sobrepeso e a obesidade prévia e/ou durante a gravidez duplica o risco dessa entidade mórbida. ${ }^{6,10,18}$

Quanto aos critérios de diagnósticos, observamos que no internamento, pacientes com PEP apresentaram labstick acima de $2+$, sugerindo que as pacientes com PEP têm maior nível de proteinúria. Já na PET, observamos duas pacientes com diagnóstico sindrômico com queixas de iminência de eclâmpsia e plaquetopenia, porém sem proteinúria no labstick.

No nosso trabalho, a PET ocorreu com maior frequência (75\%), concordando com dados da estatística mundial compulsada, que diz que o tipo de início tardio da pré-eclâmpsia compreende mais de $70 \%$ de todos os casos relatados. ${ }^{7}$

Em relação à paridade, as primíparas (123) desenvolveram mais PE, o que está de acordo com dados da literatura, que afirmam que PE é duas vezes mais comum em primíparas do que em multíparas. ${ }^{19-21}$ Dessas primíparas, 98 evoluíram com PET, aspecto esse coincidente com revisões de estudos que têm mostrado que a forma tardia é mais prevalente nas primigestas. $^{3,8,22,23}$

Mulheres com história prévia de PE têm em torno de 15 a $25 \%$ mais chance de recidiva da doença. ${ }^{19,24}$ Neste estudo, evidenciamos que nossas pacientes apresentaram $28,8 \%$ de recorrência de PE, confirmando ser este parâmetro um dado da história que não pode ser esquecido de se perguntar à paciente e anotar no prontuário. O nosso valor de recorrência foi maior do que o da literatura, possivelmente por se tratar de uma amostra de um hospital escola, referência a pacientes de alto risco da região. Nessas pacientes, o risco de desenvolver préeclâmpsia precoce foi maior que desenvolver pré-eclâmpsia tardia.

Pacientes com histórico familiar têm maior potencial de risco de desenvolver PE, o que já foi evidenciado em outros estudos que mostraram alta incidência nos familiares. $\mathrm{O}$ antecedente na mãe ou na irmã parece exercer forte influência no seu surgimento. ${ }^{8}$ Nosso estudo evidenciou apenas 12,5\% de pacientes com familiares com antecedentes de PE. Porém, por se tratar de uma investigação retrospectiva, pode ter havido omissão no tocante a essa informação, assim como não foram encontrados registros sobre os níveis pressóricos em 10 pacientes de PEP e 27 pacientes de PET no primeiro trimestre de gestação.

A hipertensão arterial foi observada em 57 pacientes desde o início da gestação, correspondendo a 27,4\% do total estudado, o que comprova, como já foi elucidado em outros estudos, que a hipertensão arterial crônica pré-gestacional é um fator de risco para desenvolvimento da PE. ${ }^{24}$

Neste estudo, não foi possível a avaliação do Doppler como fator de predição de PE, pois a quantidade dos dados obtidos foi muito reduzida. Possivelmente não realizaram o exame ou não foram documentados. Seria importante termos informações sobre este dado, anormalidades de placentação evidenciadas pela alta resistência nas artérias uterinas são consideradas em muitos estudos como importante fator preditivo de PE, especialmente do tipo PEP. O Doppler das artérias uterinas quase sempre está incluído nos modelos de análise multivariada para predição de PE. 13,22,25

Meta-análise recente tem sugerido que o uso da aspirina numa fase precoce - idade gestacional (menor que 16 semanas) - leva a uma redução no início da PE precoce e que está associada com uma redução na prevalência de morte e morbidade perinatal. ${ }^{13}$ Pelo que se pôde notar na amostra avaliada, a prevenção da pré-eclâmpsia com uso de aspirina parece pouco difundida, pois mesmo em pacientes com múltiplos fatores de risco, poucas eram as que faziam uso dessas medicações.

\section{CONCLUSÃO}

Entre os fatores de risco mais associados com PE grave, observou-se que a hipertensão arterial crônica foi o mais prevalente; e, dos fatores de risco moderado, a primiparidade, a história de doença hipertensiva em gestação anterior e o IMC elevado foram os mais encontrados. Por isso, estes fatores de risco devem compor obrigatoriamente a investigação clínica do pré-natal de todo serviço de atenção básica ou especializada.

Conclui-se também que as pacientes que iniciaram o pré-natal com níveis pressóricos elevados apresentam maior risco de desenvolver pré-eclâmpsia precoce. 


\section{REFERENCIAS}

1. Scazzocchio E, Figueras F. Contemporary prediction of preeclampsia. Curr Opin Obstet Gynecol. 2011;23(2):65-71.

2. Cnossen JS, Morris RK, ter Riet G, Mol BW, van der Post JA, Coomarasamy A, et al. Use of uterine artery Doppler ultrasonography to predict pre-eclampsia and intrauterine growth restriction: a systematic review and bivariable meta-analysis. Can Med Assoc J. 2008;178(6):701-11.

3. Laurenti R, Jorge MH, Gotlieb SL. A mortalidade materna nas capitais brasileiras: algumas características e estimativa de um fator de ajuste. Rev Bras Epidemiol. 2004;7(4):449-60.

4. Reis ZS, Lage EM, Teixeira PG, Porto LB, Guedes LR, Oliveira EC, et al. Pré-eclâmpsia precoce e tardia: uma classificação mais adequada para o prognóstico materno e perinatal? Rev Bras Ginecol Obstet. 2010;32(12):584-90.

5. Zhang J, Klebanoff MA, Roberts JM. Prediction of adverse outcomes by common definitions of hypertension in pregnancy. Obstet Gynecol. 2001;97(2):261-7.

6. Assis, TR, Viana FP, Rassi S. Estudo dos principais fatores de risco maternos nas síndromes hipertensivas da gestação. Arq Bras Cardiol. 2008;91(1):11-17.

7. Huppertz B. Placental origins of preeclampsia: challenging the current hypothesis. Hypertension. 2008;51(4):970-5.

8. von Dadelszen P, Magee LA, Roberts JM. Subclassification of preeclampsia. Hypertens Pregnancy. 2003;22(2):143-8.

9. Roberts CL, Algert CS, Morris JM, Ford JB. Increased planned delivery contributes to declining rates of pregnancy hypertension in Australia: a population-based record linkage study. BMJ Open. 2015;5(10):1-10.

10. Duley L. The global impact of pre-eclampsia and eclampsia. Semin Perinatol. 2009;33(3):130-7.

11. Duckitt K, Harrington D. Risk factors for pre-eclampsia at antenatal booking: systematic review of controlled studies. BMJ. 2005;330(7491):565.

12. Crispi F, Domínguez C, Llurba E, Martín-Gallán P, Cabero L, Gratacós E. Placental angiogenic growth factors and uterine artery Doppler findings for characterization of different subsets in preeclampsia and in isolated intrauterine growth restriction. Am J Obstet Gynecol. 2006;195(1):201-7.

13. Park F, Russo K, Williams P, Pelosi M, Puddephatt R, Walter M, et al. Prediction and prevention of early onset pre-eclampsia: impact of aspirin after first trimester screening. Ultrasound Obstet Gynecol. 2015;46(4):419-23.

14. ACOG Committee on Obstetric Practice. Practice bulletin 33: diagnosis and management of preeclampsia and eclampsia. Obstet Gynecol. 2002;99(1):159-67.

15. English FA, Kenny LC, McCarthy FP. Risk factors and effective management of preeclampsia. Integr Blood Press Control. 2015;8:7-12.

16. Ghojazadeh M, Azami-Aghdash S, Mohammadi M, Vosoogh S, Mohammadi S, Naghavi-Behzad M. Prognostic risk factors for early diagnosing of preeclampsia in nulliparas. Niger Med J. 2013;54(5):344-8.

17. Yu CK, Khouri O, Onwudiwe N, Spiliopoulos Y, Nicolaides KH; Fetal Medicine Foundation Second-Trimester Screening Group. Prediction of pre-eclampsia by uterine artery Doppler imaging: relationship to gestational age at delivery and small-for-gestational age. Ultrasound Obstet Gynecol. 2008;31(3):310-13.

18. Ness RB, Sibai BM. Shared and disparate components of the pathophysiologies of fetal growth restriction and preeclampsia. Am J Obstet Gynecol. 2006;195(1):40-9.

19. Raposo L, Ferreira C, Fernandes M, Pereira S, Moura P. Complicações da obesidade na gravidez. Arq Med. 2011;25(3):115-22.

20. Grill S, Rusterholz C, Zanetti-Dallencach R, Tercanli S, Holzgreve W, Hahn S, et al. Potential markers of preeclampsia - a review. Reprod Biol Endocrinol. 2009;7:70.

21. O'Gorman N, Wright D, Syngelaki A, Akolekar R, Wright A, Poon LC, et al. Competing risks model in screening for preeclampsia by maternal factors and biomarkers at 11-13 weeks gestation. Am J Obst Gynecol. 2016;214(1):103.e1-e12.

22. Wax JR. Risks and management of obesity in pregnancy: current controversies. Curr Opin Obstet Gynecol. 2009;21(2):117-23.

23. Alves JA, Sousa PC, Moura SB, Kane SC, Costa FS. Firsttrimester maternal ophthalmic artery Doppler analysis for prediction of pre-eclampsia. Ultrasound Obstet Gynecol. 2014;44(4):411-8.

24. Jim B, Sharma S, Kebede T, Acharya A. Hypertension in pregnancy: a comprehensive update. Cardiol Rev. 2010;18(4):17889.

25. Wright D, Syngelaki A, Akolekar R, Poon LC, Nicolaides $\mathrm{KH}$. Competing risks model in screening for preeclampsia by maternal characteristics and medical history. Am J Obstet Gynecol. 2015;213(1):62.e1-10.

26. Kleinrouweller CE, Bossuyt PM, Thilaganathan B, Vollebregt KC, Arenas Ramírez J, Ohkuchi A, et al. Value of adding second-trimester uterine artery Doppler to patient characteristics in identification of nulliparous women at increased risk for pre-eclampsia: an individual patient data meta-analysis. Ultrasound Obstet Gynecol. 2013;42(3):257-67.

\section{Como citar:}

Santos NA, Gurgel JA, Camurça CG. Avaliação dos fatores de risco maternos em gestantes admitidas com pré-eclâmpsia grave. Rev Med UFC. 2016 jul-dez;56(2):25-29. 